\title{
Fatty liver index correlates with non-alcoholic fatty liver disease, but not with newly diagnosed coronary artery atherosclerotic disease in Chinese patients
}

Zhao-Yan Jiang ${ }^{1 \dagger}$, Chen-Ying Xư ${ }^{2 \dagger}$, Xian-Xing Chang ${ }^{2}$, Wei-Wei Li ${ }^{2}$, Lu-Ying Sun ${ }^{2}$, Xiao-Bo Yang ${ }^{2}$ and Li-Fen Yu ${ }^{2 *}$

\begin{abstract}
Background: Fatty liver index (FLI) was recently established to predict non-alcoholic fatty liver disease (NAFLD) in general population, which is known to be associated with coronary artery atherosclerotic disease (CAD).

This study aims to investigate whether FLI correlates with NAFLD and with newly diagnosed CAD in a special Chinese population who underwent coronary angiography.

Methods: Patients with CAD $(n=231)$ and without CAD $(n=482)$ as confirmed by coronary angiography were included. Among them, 574 patients underwent B-ultrosonography were divided into NAFLD group $(n=209)$ and non-NAFLD group $(n=365)$. Correlation between FLI and NAFLD was analyzed using pearson's correlation. The associations between FLI and NAFLD as well as CAD were assessed using logistic regression. The predictive accuracy of FLI for NAFLD was evaluated using receiver operating characteristics (ROC) curve analysis.

Results: FLI was significantly higher in NAFLD group (37.10 \pm 1.95$)$ than in non-NAFLD group $(17.70 \pm 1.04), P<0.01$. FLI correlated with NAFLD $(r=0.372, P<0.001)$. The algorithm for FLI had a ROC-AUC of 0.721 (95\% Cl: $0.678-0.764)$ in the prediction of NAFLD. Logistic regression analysis showed that FLI was associated with NAFLD (adjusted OR $=1.038$, 95\% Cl: 1.029-1.047, P<0.01). The proportion of patients with CAD did not differ among the groups of FLI $\leq 30(32.3 \%)$, 30-60 (31.0\%), and $\geq 60$ (35.3\%). No significant association was found between FLI and CAD (adjusted OR=0.992, 95\% Cl: 0.981-1.003 in men and $\mathrm{OR}=0.987,95 \% \mathrm{Cl}: 0.963-1.012$ in women, $\mathrm{P}>0.05)$.

Conclusions: FLI showed good correlation with NAFLD in patients who underwent coronary angiography, but not with newly diagnosed CAD. This might be underestimated because some patients in non-CAD group may have other underlying cardiovascular diseases.
\end{abstract}

Keywords: Fatty liver index, Non-alcoholic fatty liver disease, Coronary artery atherosclerotic disease, Coronary angiography

\section{Background}

Non-alcoholic fatty liver disease (NAFLD) is a progressive problem in Asia-Pacific region [1]. The prevalence of NAFLD is increasing to about $15-20 \%$ in cities like Shanghai, Guangzhou and Hong Kong in China recently [2]. It is associated with metabolic disorders of lipids,

\footnotetext{
* Correspondence: gracefen@gmail.com

${ }^{\dagger}$ Equal contributors

2Department of Gastroenterology, Ruijin Hospital, Shanghai Jiao Tong University School of Medicine, Shanghai 200025, China Full list of author information is available at the end of the article
}

hypertension, diabetic mellitus and coronary arterial atherosclerotic disease (CAD). CAD is one of the leading causes of death from NAFLD [3-5]. Thus, prediction of NAFLD at earlier stage is important in prevention of the inherent process of NAFLD and the associated fatal diseases such as CAD.

So far, the diagnosis of NAFLD is based on computerized tomography (CT) scanning, magnetic resonance spectroscopy (MRS), B-ultrasonography and even liver biopsy. However, most subjects with NAFLD do not have symptoms, especially at the very early stage, which
C Biomed Central 
limits the early detection and prevention of NAFLD. Bedogni et al [6] established a formula to calculate fatty liver index (FLI) based on triglycerides, body mass index (BMI), gamma-glutamyltrasnferase (GGT) and waist circumference (WC) to predict NAFLD in Italian population. These variables are risk factors for CAD as well. Therefore, whether FLI can be used as a marker for the prediction of CAD development is worth being explored.

The aim of this study is to evaluate whether FLI is able to predict NAFLD in a special Chinese population who underwent coronary angiography and to investigate whether FLI is associated with newly diagnosed CAD.

\section{Methods}

\section{Study subjects and procedure}

This was a cross-sectional study. From January 2007 to September 2011, consecutive patients with suspected CAD (ie, those with angina or abnormal exercise stress test results) who presented for the first time for coronary angiography were recruited in this study. Patients underwent coronary angiography for reasons as congenital heart disease, congestive heart failure, cardiomyopathy and valvular heart diseases were excluded. According to criteria of National Institutes of Health Clinical Research Network [1,7], patients with alcohol intake $>140$ g/week in male or $>70 \mathrm{~g} /$ week in female were not included in the study. Patient with other causes such as viral hepatitis, drug induced hepatitis, autoimmune hepatitis, etc., were not included either. Finally, 713 patients were included in the study. In accordance with American College of Cardiology/American Heart Association guidelines, patients were defined as CAD positive (CAD group: $n=231$, male/female: $171 / 60$ ) if equal to or more than $50 \%$ diameter stenosis in any one of the major coronary arteries was found on coronary angiography. If no stenosis was observed, those patients were defined as non-CAD (nonCAD group: $n=482$, male/female: $215 / 267$ ). We did not include patients with stenosis less than $50 \%$, because these lesions may progress, and eventually, patients with no significant obstructions have significantly more cardiovascular events during follow-up than those with truly normal coronary angiograms [8].

Among 713 patients, 574 patients underwent B-type ultrasonography, who were accordingly classified into NAFLD group ( $\mathrm{n}=209$, male/female: $113 / 96)$ and nonNAFLD group ( $n=365$, male/female: 193/172). The diagnostic criteria for steatosis under B-type ultrasound was indicated by diffusely increased echogenicity ("bright") liver with liver echogenicity greater than kidney, with vascular blurring, and deep attenuation of ultrasound signal $[9,10]$. BMI was calculated by weight $/ \mathrm{height}^{2}\left(\mathrm{~kg} / \mathrm{m}^{2}\right)$. FLI was calculated by previous reported formula [6].

The study protocol was approved by Ethical Committee at Ruijin Hospital and written informed consents to participate in the study were obtained from all the patients. The study was performed in accordance with the Declaration of Helsinki.

\section{Statistical analysis}

Continuous variables were presented as means \pm S.E.M and compared by t-test between groups. Categorical variables were summarized as numbers of subjects (percentage) and compared by $\chi^{2}$ test between groups. Cochran-Armitage trend tests were also performed if there were more than two categorical variables. The predictive accuracy of FLI for NAFLD was evaluated using receiver operating characteristics (ROC) curve analysis by DeLong method. In addition, an area under the ROC curve (AUC) with $95 \%$ confidence intervals (CI) was calculated for different markers. A correlation between FLI with NAFLD was analyzed with Pearson's correlation. Associations between FLI and NAFLD as well as CAD were analyzed by logistic regression analysis, respectively. Covariates included age, gender, FLI, ALT, AST, alkaline phosphatase (ALP), total cholesterol (TC), HDL-C, LDL$\mathrm{C}$, fasting plasma glucose (FPG), history of hypertension, history of diabetic mellitus, and history of dyslipidemia. Data were expressed as odds ratio (OR) with 95\% CI were calculated. All analyses were carried out with SPSS version 17.0. The statistical significance was set at $P<0.05$. All $P$ values were two-sided.

\section{Results}

\section{The association between FLI and NAFLD}

The clinical characteristics between NAFLD and nonNAFLD groups were shown in Additional file 1: Table S1. Patients with NAFLD had significantly higher FLI $(37.10 \pm 1.95)$ than patients without NAFLD (17.70 \pm 1.04), $P<0.01$, Figure 1. According to Bedogni's 'rule in and rule out' criteria [6], we divided the patients into

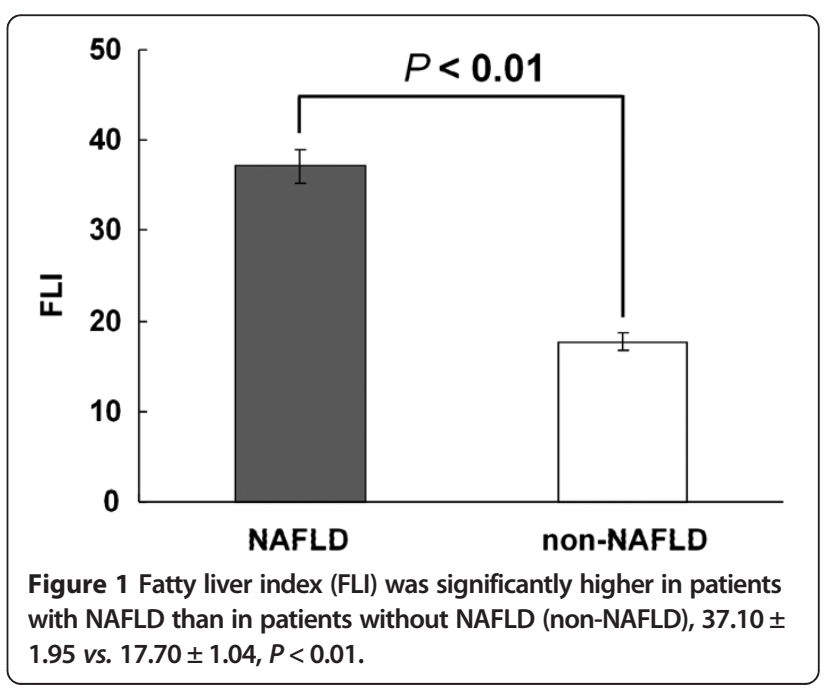


three categories: FLI $\geq 60,30<\mathrm{FLI}<60$, and $\mathrm{FLI} \leq 30$. The clinical characteristics among three FLI categories were shown in Additional file 1: Table S2. The proportion of patients with NAFLD was $74.6 \%$ (53/71) in group with FLI $\geq 60,48.3 \%$ (56/116) in group with FLI $30-60$, and $25.8 \%(100 / 387)$ in group with FLI $\leq 30$ (Cochran-Armitage trend test: $P<0.01$ ), Table 1 .

The model for FLI had a ROC-AUC value of 0.721 (95\% CI: $0.678-0.764$, Figure 2) in the prediction of NAFLD. The accuracy of FLI in the prediction of NAFLD was better than any single variable in the formula except BMI (Figure 2). Pearson's correlation showed FLI correlated with NAFLD $(r=0.372, P<$ 0.001). Using multivariate Logistic regression analysis, we found that FLI was associated with NAFLD (age-, sex-adjusted OR $=1.038,95 \%$ CI: $1.029-1.047, P<0.01$ ) (Table 2). History of dyslipidemia, diabetic mellitus, and hypertension were also factors associated with an increased risk of NAFLD (Table 2). To minimize the influence of drug therapy on the development of NAFLD, a further analysis adjusted for anti-hyperlipidemia and diabetic mellitus drugs was performed. The result showed that FLI was still associated with NAFLD (adjusted OR = 1.038, 95\% CI: 1.028-1.049, P < 0.01).

\section{Multivariate logistic regression analysis on the factors associated with CAD}

The clinical characters between CAD group and nonCAD group were shown in Additional file 1: Table S3. Unexpectedly, no significant difference was observed in the proportion of patients with CAD among groups with $\mathrm{FLI} \leq 30,30-60$ and $\geq 60$, which was $32.3 \%(156 / 483)$, $31.0 \%(45 / 145)$ and $35.3 \%$ (30/85) (Cochran-Armitage trend test: $\mathrm{P}>0.05$ ), respectively (Table 3 ).

In the multivariate Logistic regression analysis, we found that both in males and females, older age, higher total cholesterol, were common factors associated with an increased risk of CAD (Table 4). Higher HDL-C was associated with a decreased risk of CAD (Table 4). However, no significant association was found between FLI and CAD in either males or females (adjusted OR $=0.990,95 \% \mathrm{CI}$ : 0.980-1.001 in men and OR $=0.988,95 \%$ CI: $0.965-1.012$ in women, $P>0.05$ ). In this study, there were $85.5 \%$ of patients in the non-CAD group and $95.7 \%$ of patients in the

Table 1 Distribution of NAFLD and non-NAFLD subjects in different FLI categories

\begin{tabular}{ccccc}
\hline & $\begin{array}{c}\text { FLI } \leq \mathbf{3 0} \\
(\mathbf{n}=\mathbf{3 8 7})\end{array}$ & $\begin{array}{c}\text { FLI 30-60 } \\
(\mathbf{n}=\mathbf{1 1 6})\end{array}$ & $\begin{array}{c}\text { FLI } \geq \mathbf{6 0} \\
\mathbf{( n = 7 1 )}\end{array}$ & P value* \\
\hline NAFLD & $100(25.8 \%)$ & $56(48.3 \%)$ & $53(74.6 \%)$ & $<0.01$ \\
Non-NAFLD & $287(74.2 \%)$ & $60(51.7 \%)$ & $18(25.4 \%)$ & $<0.01$ \\
\hline
\end{tabular}

${ }^{*}$ Cochran-Armitage test for trend between three FLI categories.

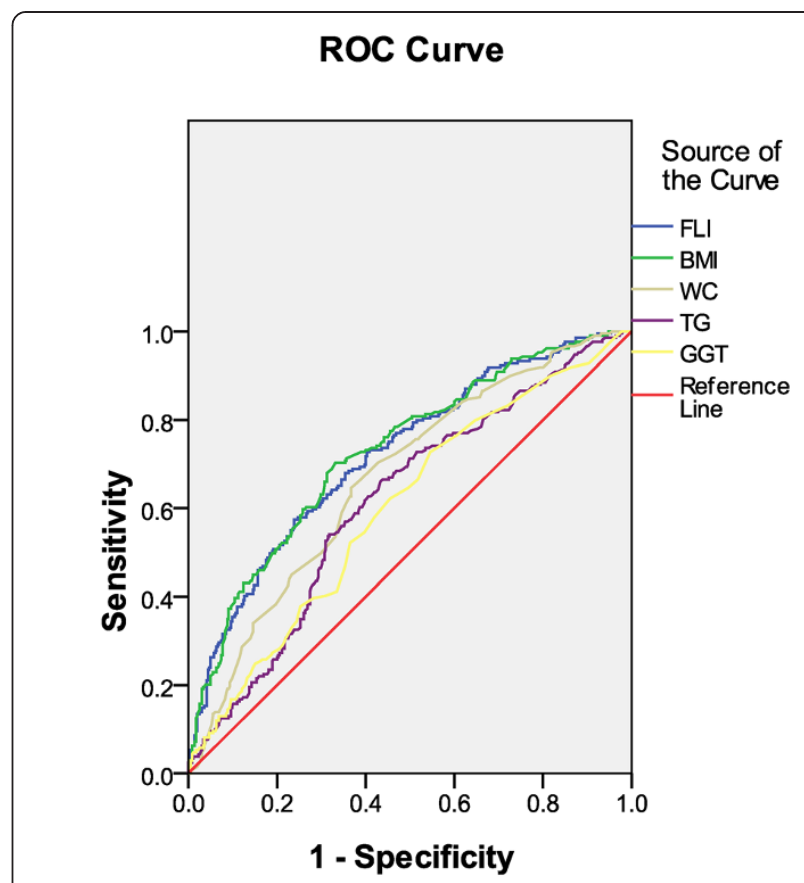

Diagonal segments are produced by ties.

\begin{tabular}{lll}
\hline Variable & Area & $95 \% \mathrm{Cl}$ \\
\hline FLI & 0.721 & $0.678-0.764$ \\
BMI & 0.728 & $0.686-0.771$ \\
WC & 0.668 & $0.623-0.713$ \\
TG & 0.616 & $0.569-0.663$ \\
GGT & 0.600 & $0.552-0.648$ \\
\hline
\end{tabular}

Figure 2 AUC-ROC of FLI for prediction of fatty liver (value $=\mathbf{0 . 7 2 1}$, 95\% Cl: 0.678-0.764). FLI, fatty liver index; AUC, area under curve; ROC: receiver operating curve.

CAD group prescribed with anti-hyperlipidemia drugs. On the other hand, $12.9 \%$ of patients in the non-CAD group and $13.4 \%$ of patients in the CAD group were treated with anti-diabetic mellitus drugs (Additional file 1: Table S3). To minimize the influence of drug therapy on the metabolism of liver, a further analysis was performed after adjusted for drug therapy. However, FLI was not associated with CAD in either gender (Table 4).

Table 2 Multivariate logistic regression analysis of factors associated with NAFLD

\begin{tabular}{lll}
\hline & OR $(\mathbf{9 5} \% \mathrm{Cl})$ & $\boldsymbol{P}$ value \\
\hline FLI & $1.038(1.029-1.047)$ & $<0.01$ \\
History of hypertension & $1.627(1.051-2.519)$ & 0.029 \\
History of diabetic mellitus & $1.890(1.190-3.003)$ & 0.007 \\
History of dyslipidemia & $1.414(1.006-2.217)$ & 0.047 \\
\hline
\end{tabular}


Table 3 Distribution of patients with and without CAD in different FLI categories

\begin{tabular}{lllll}
\hline & $\begin{array}{l}\text { FLI } \leq \mathbf{3 0} \\
(\mathbf{n}=\mathbf{4 8 3})\end{array}$ & $\begin{array}{l}\text { FLI 30-60 } \\
(\mathbf{n}=\mathbf{1 4 5})\end{array}$ & $\begin{array}{l}\text { FLI } \geq \mathbf{6 0} \\
(\mathbf{n}=\mathbf{8 5})\end{array}$ & \multicolumn{1}{c}{ value* } \\
\hline CAD $(\mathrm{n}=231)$ & $156(32.3 \%)$ & $45(31.0 \%)$ & $30(35.3 \%)$ & $>0.05$ \\
Non-CAD $(\mathrm{n}=482)$ & $327(67.7 \%)$ & $100(69.0 \%)$ & $55(64.7 \%)$ & $>0.05$
\end{tabular}

*Cochran-Armitage test for trend between three FLI categories.

\section{Discussion}

Fatty liver is usually diagnosed by CT scanning, MRS or B-ultrosonography. Although pathological diagnosis by liver biopsy is a golden standard, it is invasive and not feasible for large population study. FLI was first established by Bedogni et al [6] based on data from Italian general population. It is a simple way to calculate the possibility of fatty liver with only four parameters and reached an accuracy of 0.84 (95\% CI: 0.81-0.87). BMI, WC, TG and GGT are variables usually easy to obtain. However, due to variation of ethnicity, dietary and environmental factors, the cut-off for waist and BMI is different for the Asian people [11,12]. Thus, FLI needs to be validated when used in a different population. With the same diagnostic criteria for fatty liver disease (by ultrasonography) [6], we also showed a good correlation between FLI and non-alcoholic fatty liver disease (pearson's correlation $\mathrm{r}=0.372, \mathrm{P}<0.001$ ) in a special Chinese population who underwent coronary angiography. The accuracy of FLI was relatively lower (AUCROC value $=0.721,95 \%$ CI: $0.678-0.764$ ) than that of Bedogni et al's study, but was nearly the same as that of Korean general population (accuracy of 0.785 , 95\% CI: $0.728-0.842$ ) [13]. The results of our study provided further evidences that FLI could be considered as an easy and useful marker for screening fatty liver subjects in both general and special population.

Patients with NAFLD were more likely to have CAD as shown in both retrospective and prospective studies $[14,15]$. CAD was also the common cause of death for NAFLD in population-based cohort studies [16-18] and follow-up studies $[19,20]$. In a 13-year follow up study [21], CAD was shown to be the main cause of death in patients with NAFLD confirmed by liver biopsy. The underlying mechanism linked between NAFLD and CAD could be insulin resistance, lipid disorders, inflammation, etc [22,23]. Furthermore, NAFLD is considered as the hepatic manifestation of metabolic syndrome [23] which is believed to be a risk factor for CAD. These accumulated evidences suggested that physicians and researchers had gradually drawn attention to the relationship between NAFLD and CAD in recent years.

Based on the good correlation between FLI and NFALD in patients who underwent coronary angiography, the second aim of our study is to investigate whether FLI was related with CAD. In Calori et al's prospective study [24], they showed that FLI was associated with cardiovascular disease-related mortality and cancer-related mortality after 15-year follow-up of 2,074 Caucasian individuals in three Italian municipalities. Another study of Gastaldelli et al [25] found that increased intima media thickness, coronary heart disease risk (evaluated by Framingham risk score), and reduced insulin sensitivity were associated with high values of FLI in RISC population (also a European Caucasian population). According to International Classification of Diseases [26], total cardiovascular diseases included not only CAD but also other diseases such as disorders of the peripheral vascular system, disease of the aorta and its branches, and congenital heart disease, etc

Table 4 Multivariate logistic regression analysis of factors associated with CAD

\begin{tabular}{|c|c|c|c|c|}
\hline & \multicolumn{2}{|c|}{ Men $(n=386)$} & \multicolumn{2}{|c|}{ Women $(n=327)$} \\
\hline & OR $(95 \% \mathrm{Cl})$ & OR $(95 \% \mathrm{CI})^{\mathrm{a}}$ & OR $(95 \% \mathrm{Cl})$ & OR $(95 \% \mathrm{CI})^{\mathrm{a}}$ \\
\hline Age & $1.049(1.021-1.077)^{* *}$ & $1.047(1.019-1.075)^{* *}$ & $1.061(1.019-1.106)^{* *}$ & $1.055(1.012-1.100)^{*}$ \\
\hline Smoking & $1.404(0.760-2.593)$ & $1.390(0.748-2.582)$ & $0.759(0.122-4.729)$ & $0.750(0.120-4.689)$ \\
\hline Alcohol intake & $0.441(0.243-0.801)^{* * *}$ & $0.451(0.246-0.828)^{* *}$ & $3.384(0.416-27.532)$ & $2.858(0.357-22.863)$ \\
\hline History of hypertension & $1.289(0.714-2.328)^{*}$ & $1.079(0.579-2.011)$ & $2.774(0.989-7.779)$ & $1.850(0.638-5.359)$ \\
\hline History of dyslipidemia & $2.034(1.169-3.538)^{*}$ & $1.996(1.142-3.489)^{*}$ & $1.494(0.718-3.105)$ & $1.656(0.781-3.512)$ \\
\hline History of DM & $0.379(0.163-0.883)$ & $0.419(0.146-1.206)$ & $0.612(0.237-1.581)$ & $0.925(0.231-3.710)$ \\
\hline ALP & $1.000(0.986-1.015)$ & $0.999(0.984-1.014)$ & $0.997(0.981-1.014)$ & $0.996(0.979-1.013)$ \\
\hline $\mathrm{TC}$ & $1.745(1.393-2.185)^{* *}$ & $1.752(1.395-2.200)^{* *}$ & $1.292(1.007-1.658)^{*}$ & $1.293(1.005-1.664)^{*}$ \\
\hline $\mathrm{HDL}$ & $0.275(0.097-0.785)^{*}$ & $0.305(0.104-0.894)^{*}$ & $0.191(0.051-0.710)^{*}$ & $0.196(0.052-0.742)^{*}$ \\
\hline FPG & $1.282(1.052-1.563)^{*}$ & $1.264(1.036-1.543)^{*}$ & $1.224(0.981-1.526)$ & $1.348(1.050-1.731)^{*}$ \\
\hline FLI & $0.990(0.980-1.001)$ & $0.991(0.980-1.002)$ & $0.988(0.965-1.012)$ & $0.985(0.961-1.010)$ \\
\hline
\end{tabular}

a: adjusted for anti-hyerlipidimia and diabetic mellitus drug therapy.

* represents $\mathrm{P}<0.05$ and ${ }^{* *}$ represents $\mathrm{P}<0.01$. 
(ICD-10 codes I00-I99, Q20- Q28). CAD is limited as the blockage of one or more of the coronary arteries that supply blood to the heart which usually is caused by atherosclerosis. However, no study has evaluated the relationship between FLI and CAD till now. In order to differentiate CAD with other cardiovascular disorders, we chose coronary angiography as a golden standard. Unfortunately, we could not find the correlation between FLI and CAD in either gender. Because there was no information of coronary angiography provided in the other two studies, the certain relationship between FLI and CAD in those studies could not be speculated. The patients enrolled in our study were suspicious of CAD, but not the general population. This may be one of the reasons leading to the differences in results between our study and the aforementioned two studies [24,25]. On the other hand, patients in the control group (non-CAD patients) with negative findings of coronary angiography were different from subjects in general population; some of them might have other cardiovascular diseases, which might lead to the underestimation of the relationship between FLI and CAD.

This cross-sectional study had some limitations. First, the genders in our study could not be balanced in CAD group: ratio of male to females was about 3:1. This is due to the fact that CAD is more prevalent in males. Second, CAD usually progresses for many years starting from metabolic changes which may simultaneously affect the occurrence of fatty liver. Actually, these two diseases do not progress in parallel. In this study, the patients were all diagnosed as CAD at first-time by coronary artery angiography. However, the exact duration of the disease could not be estimated. Third, majority of the patients in the study were prescribed with anti-hyperlipidemia drugs such as statin, etc. The potential effect of these drugs on both $\mathrm{CAD}$ and fatty liver disease cannot be excluded. Therefore, we performed the analysis adjusted for both antihyperlipidemia and diabetic mellitus to minimize such possibility. Finally, Neyman bias may exist to affect the results due to the nature of study design.

\section{Conclusions}

FLI showed good correlation with NAFLD in patients who underwent coronary angiography. Although we did not find a relationship between FLI with newly diagnosed CAD in the present study, our results did not exclude the presence of correlation between FLI and cardiovascular disease which was already proved in general population $[24,25]$. With the rapid change in lifestyle, CAD becomes healthy and economic burden and one of the leading causes of death in Chinese. Recently, a cross-sectional study representative sample of 46,239 Chinese adults over 20 years of age showed that the awareness, treatment, and control of high total cholesterol were $11.0 \%, 5.1 \%$, and
$2.8 \%$, respectively [27]. This indicates that a large subpopulation would be susceptible to the development of CAD. The certain relationship between FLI and CAD is still awaited to be further clarified by the results of some large, prospective studies in Chinese population in the future.

\section{Additional file}

\begin{abstract}
Additional file 1: Fatty liver index correlates with non-alcoholic fatty liver disease, but not with newly diagnosed coronary artery atherosclerotic disease in Chinese patients. Table S1 Clinical

characteristics between NAFLD group and non-NAFLD group. Table S2 Clinical characteristics among different FLI categories*. Table S3 Clinical characteristics between CAD group and non-CAD group.
\end{abstract}

\section{Competing interests}

The authors declare that they have no competing interests.

\section{Authors' contributions}

$Z Y J$ and LFY designed the study and wrote the manuscript. ZYJ, CYX and LFY analyzed the data. CYX, XXC, WWL, LYS and XBY collected the data. All authors were involved in editing the manuscript. All authors read and approved the final manuscript.

\section{Acknowledgement}

This study was partly supported by grants from National Natural Science Foundation of China (No 81070367 and No 30872973).

\section{Author details}

${ }^{1}$ Department of Surgery, Shanghai Institute of Digestive Surgery, Shanghai 200025, China. ²Department of Gastroenterology, Ruijin Hospital, Shanghai Jiao Tong University School of Medicine, Shanghai 200025, China.

Received: 8 November 2012 Accepted: 20 June 2013

Published: 8 July 2013

\section{References}

1. Chitturi S, Farrell GC, Hashimoto E, Saibara T, Lau GK, Sollano JD: Non-alcoholic fatty liver disease in the Asia-Pacific region: definitions and overview of proposed guidelines. J Gastroenterol Hepatol 2007, 22(6):778-787.

2. Fan JG, Farrell GC: Epidemiology of non-alcoholic fatty liver disease in China. J Hepatol 2009, 50(1):204-210.

3. de Alwis NM, Day CP: Non-alcoholic fatty liver disease: the mist gradually clears. J Hepatol 2008, 48(Suppl 1):S104-S112.

4. Farrell GC, Larter CZ: Nonalcoholic fatty liver disease: from steatosis to cirrhosis. Hepatology 2006, 43(2 Suppl 1):S99-S112.

5. Vuppalanchi R, Chalasani N: Nonalcoholic fatty liver disease and nonalcoholic steatohepatitis: selected practical issues in their evaluation and management. Hepatology 2009, 49(1):306-317.

6. Bedogni G, Bellentani S, Miglioli L, Masutti F, Passalacqua M, Castiglione A, Tiribelli C: The Fatty Liver Index: a simple and accurate predictor of hepatic steatosis in the general population. BMC Gastroenterol 2006, 6:33.

7. Fan JG, Saibara T, Chitturi S, Kim Bl, Sung JJ, Chutaputti A: What are the risk factors and settings for non-alcoholic fatty liver disease in Asia-Pacific? J Gastroenterol Hepatol 2007, 22(6):794-800.

8. Emond M, Mock MB, Davis KB, Fisher LD, Holmes DR Jr, Chaitman BR, Kaiser GC, Alderman E, Killip T 3rd: Long-term survival of medically treated patients in the Coronary Artery Surgery Study (CASS) Registry. Circulation 1994, 90(6):2645-2657.

9. Saverymuttu SH, Joseph AE, Maxwell JD: Ultrasound scanning in the detection of hepatic fibrosis and steatosis. Br Med J (Clin Res Ed) 1986, 292(6512):13-15.

10. Joseph AE, Saverymuttu SH, Al-Sam S, Cook MG, Maxwell JD: Comparison of liver histology with ultrasonography in assessing diffuse parenchymal liver disease. Clin Radiol 1991, 43(1):26-31.

11. Expert WHO: Consultation. Appropriate body-mass index for Asian populations and its implications for policy and intervention strategies. Lancet 2004, 363(9403):157-163. 
12. Alberti KG, Eckel RH, Grundy SM, Zimmet PZ, Cleeman Jl, Donato KA, Fruchart JC, James WP, Loria CM, Smith SC Jr: Harmonizing the metabolic syndrome: a joint interim statement of the International Diabetes Federation Task Force on Epidemiology and Prevention; National Heart, Lung, and Blood Institute; American Heart Association; World Heart Federation; International Atherosclerosis Society; and International Association for the Study of Obesity. Circulation 2009, 120(16):1640-1645.

13. Kim JH, Kwon SY, Lee SW, Lee CH: Validation of fatty liver index and lipid accumulation product for predicting fatty liver in Korean population. Liver Int 2011, 31(10):1600-1601.

14. Lin YC, Lo HM, Chen JD: Sonographic fatty liver, overweight and ischemic heart disease. World J Gastroenterol 2005, 11(31):4838-4842.

15. Villanova N, Moscatiello S, Ramilli S, Bugianesi E, Magalotti D, Vanni E, Zoli $M$, Marchesini G: Endothelial dysfunction and cardiovascular risk profile in nonalcoholic fatty liver disease. Hepatology 2005, 42(2):473-480.

16. Dunn W, Xu R, Wingard DL, Rogers C, Angulo P, Younossi ZM, Schwimmer JB: Suspected nonalcoholic fatty liver disease and mortality risk in a population-based cohort study. Am J Gastroenterol 2008, 103(9):2263-2271.

17. Ruttmann E, Brant LJ, Concin H, Diem G, Rapp K, Ulmer H: Gammaglutamyltransferase as a risk factor for cardiovascular disease mortality: an epidemiological investigation in a cohort of 163,944 Austrian adults. Circulation 2005, 112(14):2130-2137.

18. Lee DS, Evans JC, Robins SJ, Wilson PW, Albano I, Fox CS, Wang TJ, Benjamin EJ, D'Agostino RB, Vasan RS: Gamma glutamyl transferase and metabolic syndrome, cardiovascular disease, and mortality risk: the Framingham Heart Study. Arterioscler Thromb Vasc Biol 2007, 27(1):127-133.

19. Matteoni CA, Younossi ZM, Gramlich T, Boparai N, Liu YC, McCullough AJ: Nonalcoholic fatty liver disease: a spectrum of clinical and pathological severity. Gastroenterology 1999, 116(6):1413-1419.

20. Adams LA, Lymp JF, St Sauver J, Sanderson SO, Lindor KD, Feldstein A, Angulo P: The natural history of nonalcoholic fatty liver disease: a population-based cohort study. Gastroenterology 2005, 129(1):113-121.

21. Rafig N, Bai C, Fang Y, Srishord M, McCullough A, Gramlich T, Younossi ZM: Long-term follow-up of patients with nonalcoholic fatty liver. Clin Gastroenterol Hepatol 2009, 7(2):234-238.

22. Targher G, Arcaro G: Non-alcoholic fatty liver disease and increased risk of cardiovascular disease. Atherosclerosis 2007, 191(2):235-240.

23. Kotronen $\mathrm{A}$, Yki-Jarvinen $\mathrm{H}$ : Fatty liver: a novel component of the metabolic syndrome. Arterioscler Thromb Vasc Biol 2008, 28(1):27-38.

24. Calori G, Lattuada G, Ragogna F, Garancini MP, Crosignani P, Villa M, Bosi E, Ruotolo G, Piemonti L, Perseghin G: Fatty liver index and mortality: the Cremona study in the 15th year of follow-up. Hepatology 2011, 54(1):145-152.

25. Gastaldelli A, Kozakova M, Hojlund K, Flyvbjerg A, Favuzzi A, Mitrakou A, Balkau B: Fatty liver is associated with insulin resistance, risk of coronary heart disease, and early atherosclerosis in a large European population. Hepatology 2009, 49(5):1537-1544.

26. Roger VL, Go AS, Lloyd-Jones DM, Adams RJ, Berry JD, Brown TM, Carnethon MR, Dai S, de Simone G, Ford ES, et al: Heart disease and stroke statistics-2011 update: a report from the American Heart Association. Circulation 2011, 123(4):e18-e209.

27. Yang W, Xiao J, Yang Z, Ji L, Jia W, Weng J, Lu J, Shan Z, Liu J, Tian H, et al: Serum Lipids and Lipoproteins in Chinese Men and Women. Circulation 2012, 125(18):2212-2221

doi:10.1186/1471-230X-13-110

Cite this article as: Jiang et al:: Fatty liver index correlates with nonalcoholic fatty liver disease, but not with newly diagnosed coronary artery atherosclerotic disease in Chinese patients. BMC Gastroenterology 2013 13:110

\section{Submit your next manuscript to BioMed Central and take full advantage of:}

- Convenient online submission

- Thorough peer review

- No space constraints or color figure charges

- Immediate publication on acceptance

- Inclusion in PubMed, CAS, Scopus and Google Scholar

- Research which is freely available for redistribution 